\title{
Editorial
}

\section{Treatment burden is important to patients but often overlooked by clinicians}

The focus of this issue of Breathe is "treatment burden", which is the work that patients must do to implement medical treatments, for example, taking medications, going to medical appointments, making lifestyle changes (diet, exercise, smoking cessation, etc.) and the effect of this workload on patient functioning and wellbeing [1]. The treatment workload associated with managing a respiratory condition can be substantial [2-6]. Treatment burden is distinct from "disease burden", which describes the morbidity and mortality associated with a disease. In this issue of Breathe we hear from patients with different respiratory conditions [7-9] as well as from carers [10] about the challenges associated with their healthcare workload. Their accounts highlight the importance of healthcare professionals working together with patients and carers to reduce treatment burden.

If the treatment workload goes beyond a patient's capacity, nonadherence to treatment is often the consequence. Causes can be a lack of patient understanding, for example due to treatment complexity or a patient's cognitive impairment, forgetfulness, difficulties in accessing the prescribed treatment due to cost or need to travel to a pharmacy, or practical issues such as difficulties opening medicine bottles or swallowing pills. Traditionally, clinicians have focused on increasing capacity in this situation, mainly through education. While it is important to provide patients with information that they can understand, adjusting for health literacy and using language interpreters if indicated, sometimes a reduction of treatment burden, for example through de-prescribing of medications, may be the most appropriate course of action for an individual patient. In the article "Discussing treatment burden" the authors give helpful tips on how to consider and discuss aspects of treatment burden in the patient-clinician encounter [11].

Considerations about treatment burden are ideally integrated into a shared decision-making process between clinician and patient/carer, in which the advantages and disadvantages of a treatment are weighed against each other [12]. Unfortunately, clinicians usually do not quantify the potential benefit of a treatment when talking to patients, for example by pointing out how many persons out of 100 treated persons will experience a specific positive outcome, or the average improvement than can be expected with treatment. Instead, clinicians frequently make vague statements such as "this inhaler will reduce the risk of flare ups". A major reason for this is probably that most clinicians do not memorise information on quantitative treatment benefits. Decision aids with written information for use during the clinical encounter can overcome this barrier. Quantitative information on treatment benefits will enable patients to make informed decisions about whether they are willing to accept therapies with a substantial treatment burden.

The article on "Treatment burden and ability to work" in this issue explores how the healthcare

@ERSpublications

The latest issue of Breathe focuses on "treatment burden" and the effects of this workload on patients: read the introductory editorial by Chief Editor @ClaudiaCDobler https://bit.ly/3djgNeO

Cite as: Dobler CC. Treatment burden is important to patients but often overlooked by clinicians. Breathe 2021; 17: 210031.

\section{(1) \\ CrossMark}


system, the workplace and the state can support working age people with chronic health conditions or disabilities to remain employed [13].

I would like to thank the European Lung Foundation for making a major contribution to this issue of Breathe by working closely together with patients and carers on a number of articles.

The following quote originated in the context of design thinking and customer experience, but is also relevant to a patient-centred healthcare approach that includes considerations of treatment burden:

We spend a lot time designing the bridge, but not enough time thinking about the people who are crossing it.

Dr. Prabhjot Singh

\section{Affiliations}

\section{Claudia C. Dobler ${ }^{1,2}$}

${ }^{1}$ The George Institute for Global Health, University of New South Wales, Sydney, NSW, Australia. ${ }^{2}$ Dept of Respiratory and Sleep Medicine, Liverpool Hospital, Sydney, NSW, Australia.

\section{Conflict of interest}

C.C. Dobler has nothing to disclose.

\section{References}

1. Eton DT, Ramalho de Oliveira D, Egginton JS, et al. Building a measurement framework of burden of treatment in complex patients with chronic conditions: a qualitative study. Patient Relat Outcome Meas 2012; 3: 39-49.

2. Sawicki GS, Sellers DE, Robinson WM. High treatment burden in adults with cystic fibrosis: challenges to disease selfmanagement. J Cyst Fibros 2009; 8: 91-96.

3. Harb N, Foster JM, Dobler CC. Patient-perceived treatment burden of chronic obstructive pulmonary disease. Int J Chron Obstruct Pulmon Dis 2017; 12: 1641-1652.

4. Spurr L. The treatment burden of long-term home noninvasive ventilation. Breathe 2021; 17: 200291.

5. Ting NCH, El-Turk N, Chou MSH, et al. Patient-perceived treatment burden of tuberculosis treatment. PLoS One 2020 15: e0241124.

6. El-Turk N, Chou MSH, Ting NCH, et al. Treatment burden experienced by patients with lung cancer. PLoS One 2021; 16: e0245492.

7. Maetz J, Denning J. The treatment burden of cystic fibrosis: a day-to-day experience with treatment as someone with cystic fibrosis. Breathe 2021; 17: 210013.
8. Williams C, Flewett R, Salgueiro B. Lung transplantation: the patient view from either side of the operating table. Breathe 2021; 17: 200305

9. Wilkens M, Bartlett M, Willersinn F, et al. The patient perspective of alpha-1 antitrypsin deficiency: disease burden and unmet needs. Breathe 2021; 17: 200340.

10. Denning J, Carter P, Galvin L, et al. How treatment burden affects the carer: the experiences of three individuals, a mother, a daughter and a husband. Breathe 2021; 17: 200327.

11. Powell P, Saggu R, Jones $S$, et al. Discussing treatment burden. Breathe 2021; 17: 200284

12. Dobler CC, Harb N, Maguire CA, et al. Treatment burden should be included in clinical practice guidelines. BMJ 2018; 363: k4065

13. Trakoli A. Treatment burden and ability to work. Breathe 2021; 17: 210004 